\title{
Avaliação dos hormônios sexuais e da prolactina em pacientes portadores de psoríase em placa atendidos em serviço de referência do estado do Pará
}

\author{
Evaluation of sexual hormones and prolactin in patients with plaque psoriasis attended on \\ a reference center on the state of Pará
Evaluación de hormonas sexuales y prolactina en pacientes con cuidado de psoriasis en placa asistidos en el servicio de referencia del estado de Pará

Marina Crespo Soares ${ }^{1 *}$, Amanda Maués Ramos ${ }^{1}$, Carlos Victor da Silva Nascimento ${ }^{1}$, João Victor Pereira Assunção¹, Francisco Ipslon Terezo Rosas Júnior², Francisca Regina Oliveira Carneiro¹.

\section{RESUMO}

Objetivo: Avaliar os níveis circulantes dos hormônios sexuais e da prolactina em pacientes com psoríase em comparação com controles saudáveis e determinar a correlação entre os níveis desses hormônios e a gravidade da doença. Métodos: Os níveis de estrogênio, progesterona, testosterona, hormônio folículo estimulante (FSH), hormônio luteinizante (LH) e prolactina (PRL) foram medidos em 40 pacientes com psoríase e 20 controles saudáveis. Resultados: Observou-se que os pacientes com psoríase apresentaram maiores níveis séricos de $\mathrm{FSH}$ e $\mathrm{LH}$ e menores de testosterona e estrogênio em relação aos controles saudáveis, embora essa diferença não tenha sido estatisticamente significativa. Não houve diferenças nos níveis de progesterona entre os 2 grupos avaliados. No entanto, os níveis séricos de prolactina foram significativamente maiores entre os pacientes com psoríase quando comparado aos controles saudáveis. Além disso, uma correlação positiva estatisticamente significante foi encontrada entre a prolactina e o PASI (Índice de gravidade da psoríase por área). Conclusão: Nesse sentido, infere-se que a prolactina possa desempenhar um papel na etiopatogenia da psoríase. No entanto, estudos de seguimento a longo prazo são necessários para validar a importância dessas associações.

Palavras-chave: Psoríase, Hormônio sexual, Prolactina.

\begin{abstract}
Objective: To evaluate the circulating levels of sex hormones and prolactin in patients with psoriasis in comparison with healthy controls and sought to determine the correlation between the levels of these hormones and the severity of the disease. Methods: Levels of estrogen, progesterone, testosterone, follicle stimulating hormone $(F S H)$, luteinizing hormone $(\mathrm{LH})$ and prolactin $(\mathrm{PRL})$ were measured in 40 patients with psoriasis and 20 healthy controls. Results: It was observed that patients with psoriasis had higher serum levels of FSH and LH and lower levels of testosterone and estrogen compared to healthy controls, although this difference was not statistically significant. There were no differences in progesterone levels between the 2 groups evaluated. However, serum prolactin levels were significantly higher among patients with psoriasis when compared to healthy controls. In addition, a statistically significant positive correlation was found between prolactin and PASI (Psoriasis Area Severity Index). Conclusion: In this sense, it is inferred that prolactin may play a role in the etiopathogenesis of psoriasis. However, long-term follow-up studies are needed to validate the importance of these associations.
\end{abstract}

Key words: Psoriasis, Sexual hormones, Prolactin.

1 Universidade do Estado do Pará (UEPA), Belém-PA. *E-mail: marina_c.soares@hotmail.com

2 Universidade Federal do Pará (UFPA), Belém-PA 


\section{RESUMEN}

Objetivo: Evaluar los niveles circulantes de hormonas sexuales y prolactina en pacientes con psoriasis en comparación con controles controlados y determinar la correlación entre estos niveles hormonales y la gravedad de la enfermedad. Métodos: Se midieron los niveles de estrógeno, progesterona, testosterona, hormona folículo estimulante (FSH), hormona luteinizante (LH) y prolactina (PRL) en 40 pacientes con psoriasis y 20 controles. Resultados: Tenga en cuenta que los pacientes con psoriasis tienen niveles más altos de FSH y LH y niveles más bajos de testosterona y estrógeno en comparación con los controles, aunque esta diferencia no fue estadísticamente significativa. No hay diferencias en los niveles de progesterona entre los 2 grupos que se aplican. Sin embargo, los niveles de prolactina en suero fueron significativamente mayores entre los pacientes con psoriasis en comparación con los controles recomendados. Además, se encontró una correlación positiva estadísticamente significativa entre prolactina y PASI (Índice de la Severidad del Área de Psoriasis). Conclusión: En este sentido, infiera que la prolactina puede desempeñar un papel en la etiopatogenia de la psoriasis. Sin embargo, se necesitan estudios de seguimiento a largo plazo para validar la importancia de estas asociaciones.

Palabras clave: Psoriasis, Hormonas sexuales, Prolactina.

\section{INTRODUÇÃO}

A psoríase tem distribuição universal acometendo igualmente homens e mulheres e cerca de $1 \%$ da população geral (SBD, 2012). É uma doença crônica poligenética e inflamatória da pele, com caráter autoimune, que se apresenta de diversas formas tais como gutata, pustular e palmoplantar, sendo a forma vulgar (PV) é o tipo mais comum (ENGIN B, et al., 2017; PIETRZAK A, et al., 2014). A clínica é representada por lesões eritematoescamosas que acometem áreas como cotovelos e joelhos ou toda a pele (RAPOSO I e TORRES T, 2016; TOOSSI P et al, 2015).

A etiopatogênese da PV inclui a inflamação crônica da derme e da epiderme devido a invasão e a ativação de células imunológicas, especialmente linfócitos T helper 17 (Th17) e T helper 1 (Th1) e modificações na diferenciação dos queratinócitos que resultam em hiperproliferação (HUSAKOVA M et al, 2015; GOTTLIEB A et al, 2017; SAAD F et al, 2016).

Nesse sentido, alguns hormônios podem ter papel importante na patogênese e na gravidade da psoríase devido aos seus efeitos sobre a proliferação de queratinócitos, de células epiteliais e de linfócitos e a uma provável influência sobre a reação inflamatória local na pele (HAU CS, et al., 2014). Particularmente, os hormônios esteroides e a prolactina estão envolvidos nesses processos (TOOSSI P, et al., 2015; KEEN MA e HASSAN I, 2014).

A hipótese de que os hormônios sexuais e a prolactina possam agir como desencadeantes ou moduladores da evolução da psoríase é sustentada por alguns estudos que demostraram a expressão da doença em diferentes fases hormonais da vida, observando um agravamento desta na puberdade, na menopausa e no pós-parto, situações em que são visualizados menores níveis dos hormônios gonadais (MURASE JE, et al., 2005).

Dessa forma, dada a escassez de dados disponíveis na literatura sobre o papel dos hormônios sexuais e da prolactina na etiopatogenia da psoríase e sabendo-se da real importância de elucidar os mecanismos que influem na gênese desta doença, busca-se com o presente estudo avaliar os níveis circulantes dos hormônios sexuais e da prolactina em pacientes com psoríase em comparação com os controles saudáveis e determinar a correlação entre os níveis desses hormônios e a gravidade da doença.

\section{MÉTODOS}

Trata-se de um estudo de caso-controle analítico prospectivo, realizado no período correspondente entre janeiro 2018 e janeiro de 2019 no Serviço de Dermatologia da Universidade do Estado do Pará (UEPA). 
O protocolo do estudo foi elaborado de acordo com a declaração de Helsinque e o código de Nuremberg, respeitadas as Normas de Pesquisa Envolvendo Seres Humanos (Resolução do Conselho Nacional de Saúde 66/12) do Conselho Nacional de Saúde e implementado após receber a aprovação do comitê de ética da universidade do estado do Pará (número do parecer de aprovação: 2.750.764). Após o consentimento informado por escrito, os pacientes e controles foram selecionados para elegibilidade.

Foram incluídas no estudo 40 pacientes com psoríase (19 mulheres e 21 homens; idade média 51,7 $\pm 16,3$ anos). O grupo controle foi composto por 20 voluntários saudáveis, pareados por idade e sexo ( 9 mulheres, 11 homens, idade média 49,3 $\pm 13,79$ anos), os quais não possuíam qualquer história pessoal ou familiar de psoríase.

Em ambos os grupos, os participantes com as seguintes condições foram excluídos do estudo: gravidez ou lactação, anormalidades menstruais, terapia hormonal atual, consumo de medicamentos que afetem os níveis séricos de prolactina (antidepressivos, antipsicóticos, butirofenonas, estrógenos, bloqueadores H2, metildopa, metoclopramida, fenotiazinas, reserpina, verapamil, etc.), prolactinoma, doenças do hipotálamo, condições estas que podem alterar os níveis dos hormônios avaliados.

O exame dermatológico completo foi feito para cada paciente para determinar a extensão e distribuição da doença. A gravidade clínica da psoríase foi avaliada usando o índice de gravidade da psoríase por área (PASI).

Após a avaliação clínica e exame dermatológico de cada paciente, foram solicitadas dosagens séricas de estrogênio, progesterona, hormônio folículo-estimulante (FSH), hormônio luteinizante (LH), testosterona e prolactina (PRL) para os 40 pacientes com psoríase e para os 20 controles pareados. Todos os níveis hormonais foram medidos por ELISA.

As informações da caracterização amostral foram apuradas em banco de dados elaborado no software Microsoft $\circledast$ Office Excel® 2016.

$\mathrm{Na}$ aplicação da Estatística Descritiva, foram construídos tabelas e gráficos para apresentação dos resultados e calculadas as medidas de posição como média aritmética e desvio padrão.

A estatística analítica foi utilizada para avaliar os resultados das variáveis categóricas da amostra através do Teste G e Teste Qui-Quadrado Aderência para tabelas univariadas e para as variáveis numéricas o Coeficiente de Correlação de Pearson.

As estatísticas descritiva e analítica foram realizadas no software BioEstat $\AA^{\circledR}$ 5.3. Para a tomada de decisão, adotou-se o nível de significância $\alpha=0,05$ ou $5 \%$, sinalizando com asterisco $\left({ }^{*}\right)$ os valores significantes.

\section{RESULTADOS}

Dos 40 pacientes com psoríase, 19 (47,5\%) eram do sexo feminino e 21 (52,5\%) eram do sexo masculino. A idade média \pm Desvio-Padrão dos pacientes foi de $51,7 \pm 16,3$, com idade mínima de 19 e máxima de 70 anos, havendo incidência significante de casos com idade superior a 50 anos.

Em virtude disso, observou-se um predomínio no estudo de mulheres menopausadas $(57,89 \%)$ em detrimento das que estavam no período fértil. Notou-se também que a maioria dos pacientes eram casados $(67,5 \%)$, possuíam escolaridade de ensino superior completo $(25,0 \%)$ e tinham como ocupação um ambiente fechado (70\%).

A duração média da psoríase foi de 15,5 $\pm 10,5$ anos, variando de 1 a 50 anos. Segundo o escore PASI (Índice de gravidade da psoríase por área), houve um predomínio significativo de doença leve, com $65 \%$ dos pacientes apresentando PASI < 10 (doença leve) e $35 \%$ apresentando PASI $\geq 10$ (doença moderada a grave). 
Tabela 1 - Perfil epidemiológico dos pacientes com psoríase.

\begin{tabular}{|c|c|c|c|}
\hline Perfil epidemiológico & Frequência & $\%(n=40)$ & p-valor \\
\hline Faixa etária (em anos) * & & & $<0.0001$ \\
\hline$<20$ & 1 & $2,5 \%$ & \\
\hline 20 a 29 & 2 & $5,0 \%$ & \\
\hline 30 a 39 & 4 & $10,0 \%$ & \\
\hline 40 a 49 & 6 & $15,0 \%$ & \\
\hline 50 a $59^{*}$ & 15 & $37,5 \%$ & \\
\hline$>=60^{*}$ & 12 & $30,0 \%$ & \\
\hline Mín / Média — DP / Máx & & $\pm 13.0 / 70$ & \\
\hline Sexo & & & 0,8744 \\
\hline Feminino & 19 & $47,5 \%$ & \\
\hline Masculino & 21 & $52,5 \%$ & \\
\hline Estado civil & & & 0,6714 \\
\hline Solteiro(a) & 13 & $32,5 \%$ & \\
\hline Casado(a) & 27 & $67,5 \%$ & \\
\hline Ocupação & & & $0.0177^{\star \star}$ \\
\hline Local Aberto & 12 & $30,0 \%$ & \\
\hline Local Fechado* & 28 & $70,0 \%$ & \\
\hline Idade fertil & & $n=20$ & 0,5027 \\
\hline Sim & 8 & $40,0 \%$ & \\
\hline Menopausadas & 12 & $60,0 \%$ & \\
\hline PASI & & & $0.0478^{\star \star}$ \\
\hline Doença leve* & 26 & $65,0 \%$ & \\
\hline Doença Moderada a Grave & 14 & $35,0 \%$ & \\
\hline Tempo de doença (anos) & & & 0,8063 \\
\hline 01 a 05 & 7 & $17,5 \%$ & \\
\hline 06 a 10 & 8 & $20,0 \%$ & \\
\hline 11 a 15 & 9 & $22,5 \%$ & \\
\hline 16 a 20 & 8 & $20,0 \%$ & \\
\hline 21 a 25 & 3 & $7,5 \%$ & \\
\hline Acima de 25 & 5 & $12,5 \%$ & \\
\hline Mín / Média \pm DP / Máx & & $\pm 10.5 / 50$ & \\
\hline Escolaridade & & & 0,4651 \\
\hline Semi analfabeto & 2 & $5,0 \%$ & \\
\hline Fundamental incompleto & 7 & $17,5 \%$ & \\
\hline Fundamental completo & 6 & $15,0 \%$ & \\
\hline Médio incompleto & 7 & $17,5 \%$ & \\
\hline Médio completo & 7 & $17,5 \%$ & \\
\hline Superior incompleto & 5 & $12,5 \%$ & \\
\hline Superior completo & 10 & $25,0 \%$ & \\
\hline
\end{tabular}

Legenda: *Teste G Aderência. ${ }^{* *}$ Teste Qui-Quadrado Aderência.

Fonte: Soares MC, et al., 2019.

Ao comparar a dosagem dos hormônios sexuais e da PRL nos grupos caso e controle, observou-se níveis mais elevados dos hormônios FSH e LH em pacientes com psoríase e níveis maiores de testosterona e estradiol no grupo controle, embora de forma não significativa. Não houve diferenças nos níveis de progesterona entre os 2 grupos avaliados. Foram encontrados níveis significativamente maiores de prolactina nos pacientes com psoríase $(19,6 \pm 17,2 \mathrm{ng} / \mathrm{ml})$ em relação ao grupo controle $(10 \pm 4,2)$, com $p<0,05$. 
Tabela 2 - Comparação das características dos pacientes com psoríase com pacientes do grupo controle.

\begin{tabular}{|c|c|c|c|c|}
\hline Variável & Caso $(n=40)$ & Controle $(n=20)$ & p-valor & \\
\hline Idade (anos) & $51.7 \pm 16.3$ & $30.1 \pm 12.9$ & $<0.0001^{*}$ & \\
\hline Estrogênio & $28.0 \pm 12.9$ & $42.5 \pm 70.4$ & $0,0833 \mathrm{pg} / \mathrm{ml}$ & $\begin{array}{l}\text { Fase folicular } 12,55 \text { a } 166 \mathrm{pg} / \mathrm{ml} \\
\text { Fase ovulatória } 85,8 \text { a } 498 \mathrm{pg} / \mathrm{ml} \\
\text { Fase lútea } 43,8 \text { a } 211 \mathrm{pg} / \mathrm{ml} \\
\text { Pós-menopausa Até } 54,7 \mathrm{pg} / \mathrm{ml} \\
\text { Masculino } 7,63 \text { a } 42,6 \mathrm{pg} / \mathrm{ml}\end{array}$ \\
\hline Progesterona & $0.3 \pm 0.9$ & $0.3 \pm 0.3$ & $0,4402 \mathrm{ng} / \mathrm{ml}$ & $\begin{array}{l}\text { Fase folicular } 0,057 \text { a } 0,893 \mathrm{ng} / \mathrm{ml} \\
\text { Fase ovulatória } 0,121 \text { a } 12,0 \mathrm{ng} / \mathrm{ml} \\
\text { Fase lútea } 1,83 \text { a } 23,9 \mathrm{ng} / \mathrm{ml} \\
\text { Pós-menopausa } 0,05 \text { a } 0,126 \mathrm{ng} / \mathrm{ml} \\
\text { Masculino } 0,05 \text { a } 0,149 \mathrm{ng} / \mathrm{ml}\end{array}$ \\
\hline
\end{tabular}

\begin{tabular}{|c|c|c|c|c|}
\hline Testosterona & $162.8 \pm 187.1$ & $176.9 \pm 252.9$ & $0,3774 \mathrm{ng} / \mathrm{dl}$ & $\begin{array}{l}\text { Homens } 20-49 \text { anos }-249-836 \\
\mathrm{ng} / \mathrm{dl} \\
\text { Homens } \geq 50 \text { anos }-193-740 \mathrm{ng} / \mathrm{dl} \\
\text { Mulheres } 20-49 \text { anos }-8,4-48,1 \\
\mathrm{ng} / \mathrm{dl} \\
\text { Mulheres } \geq 50 \text { anos } 2,9-40,8 \mathrm{ng} / \mathrm{dl}\end{array}$ \\
\hline Prolactina & $19.6 \pm 17.2$ & $10.0 \pm 4.2$ & $0.0009^{*} \mathrm{ng} / \mathrm{ml}$ & $\begin{array}{l}\text { Homens } 4,04 \text { a } 15,02 \mathrm{ng} / \mathrm{ml} \\
\text { Mulheres } 4,79 \text { a } 23,3 \mathrm{ng} / \mathrm{ml}\end{array}$ \\
\hline $\mathrm{FSH}$ & $23.8 \pm 28.9$ & $14.5 \pm 32.7$ & $0,1321 \mathrm{mUl} / \mathrm{ml}$ & $\begin{array}{l}\text { Fase folicular } 3,5 \text { a } 12,5 \mathrm{mUl} / \mathrm{ml} \\
\text { Fase ovulatória } 4,7 \text { a } 21,5 \mathrm{mUl} / \mathrm{ml} \\
\text { Fase lútea } 1,7 \text { a } 7,7 \mathrm{mUl} / \mathrm{ml} \\
\text { Pós-menopausa } 25,8 \text { a } 134,8 \\
\mathrm{mUl} / \mathrm{ml} \\
\text { Masculino } 1,5 \text { a } 12,4 \mathrm{mUl} / \mathrm{ml}\end{array}$ \\
\hline $\mathrm{LH}$ & $13.0 \pm 9.9$ & $9.9 \pm 15.1$ & $0,1726 \mathrm{mUl} / \mathrm{ml}$ & $\begin{array}{l}\text { Fase folicular 2,4 a } 12,6 \mathrm{mUl} / \mathrm{ml} \\
\text { Fase ovulatória } 14 \text { a } 95,6 \mathrm{mUl} / \mathrm{ml} \\
\text { Fase lútea } 1,0 \text { a } 11,4 \mathrm{mUl} / \mathrm{ml} \\
\text { Pós-menopausa } 7,7 \text { a } 58,5 \mathrm{mUl} / \mathrm{ml} \\
\text { Masculino } 1,7 \text { a } 8,6 \mathrm{mUl} / \mathrm{ml}\end{array}$ \\
\hline
\end{tabular}

Fonte: Soares MC, et al., 2019.

Ademais, uma correlação positiva significante foi detectada entre o PASI e os níveis séricos de prolactina (PRL) no grupo com psoríase $(p<0,05)$, o que significa que níveis maiores desse hormônio estão associados a um PASI mais elevado e, portanto, a doença mais grave. Não foram encontradas diferenças estatisticamente significativas entre os demais hormônios avaliados e o PASI. 
Tabela 3 - Correlação entre o índice de gravidade da psoríase por área (PASI) e os níveis séricos dos hormônios sexuais e da prolactina.

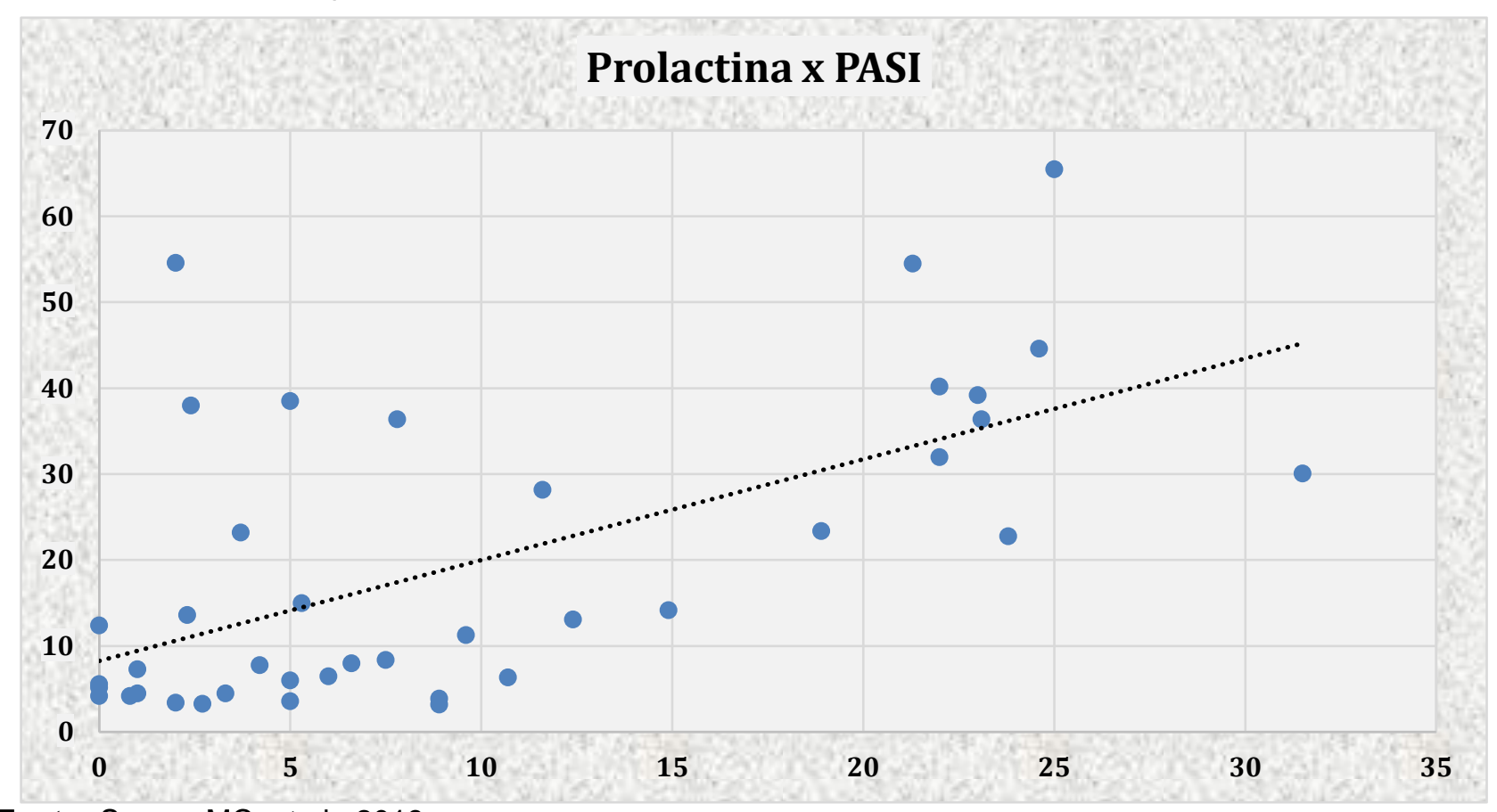

Fonte: Soares MC, et al., 2019.

\section{DISCUSSÃO}

A psoríase é uma doença da pele, crônica e imunologicamente mediada, caracterizada principalmente pelo envolvimento de linfócitos $\mathrm{T}$ tipo $\mathrm{T}$ Helper 1 e $\mathrm{T}$ Helper 17 (Th1 e Th17), tendo como principais mecanismos patogênicos a inflamação crônica e a hiperproliferação de queratinócitos (COIMBRA S, et al., 2012; SBD, 2012; WU S, et al., 2016). São muitos os fatores, endógenos e exógenos, que influenciam no surgimento, no curso e na evolução da doença, dentre eles destacam-se as variações hormonais existentes ao longo da vida, as quais podem agir como desencadeantes ou moduladoras da evolução dessa patologia (ROMAN II, et al., 2016).

A prolactina (PRL), um neuropeptídeo produzido por células lactotróficas na glândula pituitária anterior, é mais conhecida por seus efeitos na lactação e na reprodução. Porém, recentemente, uma gama de ações desse hormônio foi documentada, incluindo sua íntima conexão com a pele. Ele foi implicado como um importante imunomodulador, podendo influenciar tanto nas reações imunes humorais quanto as mediadas por células, desempenhando um papel importante na expressão de várias doenças auto-imunes, incluindo a psoríase (JARA LJ et al, 1991; DE BELLIS A, 2005).

Sabe-se que a PRL exerce efeitos imunoestimulantes que podem provocar o desenvolvimento da psoríase (KANDA N, et al., 2013): Estimula a proliferação de queratinócitos, favorece a infiltração de linfócitos Th1, estimula a produção de IFN-gama e promove a angiogênese (GIROLOMONI G, et al., 1996; DE BELLIS A, 2005, LANGAN EA, et al., 2012).

Vários estudos sugerem que a hiperprolactinemia está envolvida na etiopatogenia da psoríase. Sanchez SR e Umbert P (2000) observaram que a doença pode ser agravada na presença de um prolactinoma (microadenoma da glândula pituitária secretor de PRL). Tais pacientes obtiveram melhora da doença após terapia com bromocriptina, um potente inibidor dopaminérgico da secreção da PRL hipofisária, sugerindo que a gravidade e extensão das lesões de pele desses pacientes se correlacionavam diretamente com os níveis de PRL. Além disso, Dunna SF e Finlay AY (1989) mostraram que nos primeiros meses do pós-parto a psoríase pode piorar, refletindo a hiperprolactinema fisiológica associada a lactação. 
Concordando com tais fatos, Buskila D et al. (1991) em estudo com mulher portadora de artrite psoriásica, também demostraram remissão de lesões epidérmicas da psoríase e melhora acentuada da doença articular após tratamento com bromocriptina (fármaco agonista da dopamina usado no tratamento de tumores da hipófise, doença de Parkinson, hiperprolactinemia e outras).

No presente estudo, os níveis de PRL circulante foram consideravelmente maiores no grupo com psoríase em comparação ao grupo controle e uma correlação positiva, estatisticamente significante, foi encontrada entre os níveis desse hormônio e a gravidade da doença.

Tais resultados foram consistentes com o estudo de Giasuddin AS et al. (1998) em que se observou níveis significativamente maiores de PRL em pacientes com psoríase em relação aos controles; e com outros estudos em que foi encontrada essa mesma associação entre os níveis séricos de PRL e prolactina e deste hormônio com e a gravidade da doença. (DILMÉ-CARRERAS E, et al., 2011; KEEN MA e HASSAN I, 2014; AZIZZADEH M, et al., 2009; ABOUKHEDR NA e EID AA, 2013)

Há pesquisas que demostram não só a relação dos níveis séricos da PRL com a gravidade da doença, mas também com a duração e com o tratamento (DILMÉ-CARRERAS E, et al., 2011; KEEN MA e HASSAN I, 2014). Dilmé-Carreras $E$ et al. (2011) observaram uma redução significativa dos níveis de PRL em pacientes com psoríase após 6 semanas de tratamento com Tacalcitol (análogo da vitamina D usado no tratamento da psoríase).

Com base nesses achados, sugere-se que a PRL possa realmente desempenhar um papel importante na patogênese da doença, estando de acordo com Dilmé-Carreras E et al. (2011) e Keen MA e Hassan I (2014), que sugeriram que os níveis séricos de PRL possam servir como marcadores biológicos da atividade da doença psoriásica.

Em relação ao estrogênio, sabe-se que este influencia a resposta imunológica modulando o desenvolvimento e a ativação de células imunes, por meio do controle exercido sobre a expressão de diferentes citocinas (DONG G, et al., 2015). Em baixas doses, esse hormônio estimula a produção de citocinas pró-inflamatórias (ex. Interleucina-1, Interleucina-6, Fator de Necrose Tumoral Alfa), por promover a polarização da resposta imune para Th1. Portanto, age a favor da resposta inflamatória e imunológica. Em contrapartida, quando em doses elevadas, exerce efeito inibidor dos componentes da resposta imune, da feita que estimula a proliferação de células Th2 e a produção de citocinas a elas associadas (PENNELL LM, et al., 2012).

Segundo Murase JE et al. (2005), tal constatação pode justificar a piora da doença no período pós-parto, na menopausa e no período peri-menstrual, situações em que se observam declínio fisiológico nos níveis de estrogênio; e melhora da doença durante a gestação, quando há aumento desse hormônio. Os autores observaram que, durante a gravidez, $55 \%$ dos pacientes tiveram melhora da doença, enquanto no pós-parto $65 \%$ pioraram.

Ademais, Cemil BC et al. (2015), demostraram melhora dos sintomas da psoríase em pacientes tratadas com contraceptivos orais de estrogênio em altas doses. Desse modo, pode-se supor que baixos níveis desse hormônio estejam intimamente implicados na etiopatogenia da doença e que ele tenha um potencial papel protetor na psoríase, promovendo um estado de tolerância imunológica (CEMIL BC, et al., 2015). Nesse sentido, tal constatação pode explicar a detecção de níveis inferiores de estrogênio (embora não estatisticamente significativo) nos portadores de psoríase em relação ao grupo controle no presente estudo.

No que diz respeito a progesterona, no estudo de Murase JE et al. (2005), também foi demostrado que níveis desse hormônio isoladamente não exercem influência sobre a psoríase, estando associado a melhora da doença apenas quando associado a altos níveis de estrogênio.

Por outro lado, Roman II et al. (2016), afirma que a progesterona pode precipitar algumas formas de psoríase, sobretudo a pustular. No presente estudo não houve diferença entre os níveis de progesterona dos pacientes com psoríase e dos controles. Então, pode-se inferir uma menor associação deste com a patogênese da doença. 
Somado a isso, estudos demonstram que epiderme, derme, folículo piloso e glândulas sebáceas expressam receptores de androgênios (ROMAN II, et al., 2016; KOPERA D, 2017). Por esta razão, esses hormônios manifestam uma variedade de efeitos biológicos e imunológicos na pele (CEMIL BC, et al., 2015; ISIK S, et al., 2016), dentre eles o de antagonizar a produção macrofágica do fator de crescimento endotelial vascular (VEGF), o qual prolonga a inflamação e o processo de cicatrização de feridas (JOBE SO, et al., 2010).

Segundo Atzeni $\mathrm{F}$ et al. (2010), os andrógenos diminuem nas doenças inflamatórias crônicas, o que condiz com o que foi visualizado no presente estudo, em que os pacientes com psoríase obtiveram média menor da dosagem de testosterona, mesmo que essa diferença não tenha sido estatisticamente significativa. De forma similar, Cemil BC et al. (2015) obtiveram essa mesma diferença, porém de forma estatisticamente significante.

Embora se note uma redução dos níveis de testosterona, ainda não existem dados consistentes que indiquem ou não a terapia hormonal para esses pacientes, havendo estudos que relatam que esta pode exacerbar a psoríase (ROMAN II, et al, 2016) e outros que apontam uma melhora considerável da doença da pele com redução do PASI após administração de testosterona (SAAD F, et al, 2016).

Em outra análise, fisiologicamente, pode-se observar níveis de hormônio folículo-estimulante (FSH) ligeiramente elevados durante a fase folicular precoce do ciclo menstrual (MULLIGAN EM et al., 2019). No seu estudo, Tuğrul Ayanoğlu B et al. (2018) avaliou as reservas ovarianas em pacientes femininas em idade reprodutiva, durante a fase folicular precoce (dias 2 a 4) do ciclo menstrual, não grávidas e com psoríase. 0 autor identificou níveis consideravelmente mais altos, estatisticamente significativos $(p<0,05)$, de FSH e da relação $\mathrm{FSH} / \mathrm{LH}$ (hormônio luteinizante) nessas pacientes em comparação aos controles saudáveis. Nesse sentido, o FSH pode estar diretamente relacionado à doença.

Concordando com achado citado, o presente estudo identificou resultados semelhantes, embora não estatisticamente significativos. Contudo, a prevalência de menopausadas na amostra deste estudo pode ser um possível viés de comparação.

\section{CONCLUSÃO}

Tendo em vista os resultados do presente estudo pode-se concluir que os níveis séricos de prolactina foram significativamente aumentados entre os pacientes com psoríase em relação aos pacientes controle. A PRL teve correlação positiva com o PASI. Além disso, não se observou diferença significativa entre os níveis séricos de estrogênio, testosterona, progesterona, FSH e LH entre os pacientes com psoríase em relação aos pacientes controle e nem correlação significante desses hormônios com a gravidade da doença. Esses dados devem ser considerados preliminares por natureza. Estudos de seguimento a longo prazo são necessários para confirmar a importância dessas associações em uma população maior e mais diversificada. No entanto, o presente estudo sugere que a PRL desempenha um papel significativo na patogênese da psoríase, podendo servir como marcador biológico da atividade da doença e ser no futuro importante alvo terapêutico das drogas que atuam no controle da doença.

\section{REFERÊNCIAS}

1. ABOUKHEDR NA, EID AA. Clinical efficacy of bromocriptine and the influence of serum prolactin levels on disease severity in patients with chronic plaque-type psoriasis. Alexandria J Med. 2013; 49:385-389. [DOI: http://dx.doi.org/10.1016/j.ajme.2013.04.001].

2. ATZENI F, et al. Psoriatic arthritis: clinical improvement and correlation with hormone axes in etanercept-treated patients. Ann N Y Acad Sci. 2010; 1193:176- 178. [PMID: 20398026].

3. AZIZZADEH M, et al. Does Prolactin Indicate Severity of Psoriasis? Iran J Dermatol, 2009;12(3):79-81.

4. BUSKILA D, et al. Improvement of psoriatic arthritis in a patient treated with bromocriptine for hyperprolactinaemia. $J$ Rheumatol. 1991 Apr;18(4):611-2. [PMID: 2066954]. 
5. CEMIL BC, et al. Sex hormones in male psoriasis patients and their correlation with the Psoriasis Area and Severity Index. J Dermatol. 2015 May;42(5):500-3. [PMID: 25728622].

6. COIMBRA S, et al. The roles of cells and cytokines in the pathogenesis of pso riasis. Int $\mathrm{J}$ Dermatol. 2012 Apr;51(4):389-95. [PMID: 22435425].

7. DE BELLIS A, et al. Prolactin and autoimmunity. Pituitary. 2005;8(1):25-30. [PMID: 16411065]

8. DILMÉ-CARRERAS E, et al. Serum prolactin levels in psoriasis and correlation with cutaneous disease activity. Clin Exp Dermatol. 2011 Jan;36(1):29-32. [PMID: 20608944].

9. DONG G, et al. $17 \beta$-estradiol contributes to the accumulation of myeloid-derived suppressor cells in blood by promoting TNF- $\alpha$ secretion. Acta Biochim Biophys Sin (Shanghai). 2015;47(8):620-629. [PMID: 26071573].

10. DUNNA SF e FINLAY AY. Psoriasis: Improvement during and worsening after pregnancy. Br J Dermatol 1989 Apr;120(4):584. [PMID:2730848].

11. ENGIN B, et al. Palmoplantar psoriasis. Clinics in Dermatology (2017) 35, 19-27. [DOI: https://doi.org/10.1016/j.clindermatol.2016.09.004]

12. GIASUDDIN AS, et al. Prolactin: does it have a role in the pathogenesis of psoriasis? Dermatology. 1998;197(2):11922. [PMID: 9732158].

13. GIROLOMONI G, et al. Prolactin stimulates proliferation of cultured human keratinocytes. J Invest Dermatol. 1993. Sep;101(3):275-9. [PMID: 8370964].

14. GOTTLIEB A, et al. Secukinumab shows significant efficacy in palmoplantar psoriasis: Results from GESTURE, a randomized controlled trial. J AM Acad Dermatol. 2017 Jan;76(1):70-80. [PMID: 27707593].

15. HAU CS, et al. Prolactin induces the production of Th17 and Th1 cytokines/chemokines in murine Imiquimod-induced psoriasiform skin. JEADV 2014 Oct;28(10):1370-9. [PMID: 24304413]

16. HUSAKOVA M, et al. Elevated serum prolactin levels as a marker of inflammatory arthritis in psoriasis vulgaris. Biomed Pap Med Fac Univ Palacky Olomouc Czech Repub. 2015 Dec;159(4):562-8. [PMID: 26175050].

17. ISIK S, et al. A Review on the Link between Psoriasis Vulgaris and Polycystic Ovary Syndrome. International Journal of Gynecology, Obstetrics and Neonatal Care, 2016, Vol. 3, No. 1. [DOl: 6 http://dx.doi.org/10.15379/24089761.2016.03.01.02].

18. JARA LJ, et al. Prolactin, immunoregulation, and autoinmune diseases. Semin Arthritis Rheum 1991; 20:273-84. [DOI: https://doi.org/10.1016/0049-0172(91)90028-X]

19. JOBE SO, et al. Estradiol-17beta and its cytochrome P450- and catechol-O- methyltransferase-derived metabolites stimulate proliferation in uterine artery endothelial cells: role of estrogen receptor-alpha versus estrogen receptor-beta. Hypertension. 2010;55(4):1005- 1011. [PMID: 20212268].

20. KANDA N, et al. Prolactin May Promote the Development of Psoriasis: Reawakened Issue. J Clin Exp Dermatol. 2013. Res 4:198. [DOI: 10.4172/2155-9554.1000198].

21. KEEN MA e HASSAN I. Serum prolactin levels in psoriasis and its association with disease activity: A case-control study. Indian J Dermatol 2014; 59:562-6. [DOI: 10.4103/0019-5154.143512].

22. KOPERA, D. Impact of Testosterone on Hair and Skin. Endocrinol Metab Synd 2015, 4:3. [DOI: 10.4172/21611017.1000187].

23. LANGAN EA, et al. Exploring the role of prolactin in psoriasis. Arch Dermatol Res. 2012 Mar;304(2):115-8. [PMID: 22249743]

24. MULLIGAN EM, et al. Effects of menstrual cycle phase on associations between the error-related negativity and checking symptoms in women. Psychoneuroendocrinology 103 (2019) 233-240. [PMID: 30721837].

25. MURASE JE, et al. Hormonal effect on psoriasis in pregnancy and post-partum. Arch Dermatol. 2005 May;141(5):6016. [PMID: 15897382].

26. PENNELL LM, et al. Sex affects immunity. J Autoim- mun 2012; 38: 282-291. [PMID: 22225601].

27. PIETRZAK A, et al. Eruption of palmoplantar pustular psoriasis in patient treated with anti-androgen therapy for prostate cancer and aggravation of lesions after statin treatment. Cent. Eur. J. Med. (2014) 9: 657. [DOI: https://doi.org/10.2478/s11536-013-0310-2]

28. RAPOSO I e TORRES T. Palmoplantar Psoriasis and Palmoplantar Pustulosis: Current Treatment and Future Prospects. Am J Clin Dermatol 2016 Aug;17(4):349-58. [PMID: 27113059].

29. ROMAN II, et al. The role of hormones in the pathogenesis of psoriasis vulgaris. Clujul Med. 2016;89(1):11-8. [PMID: 27004020]. 
30. SAAD F, et al. Hypogonadal men with psoriasis benefit from long-term testosterone replacement therapy - a series of 15 case reports. Andrologia 2016 Apr;48(3):341-6. [PMID:26184426].

31. SAAD F, et al. Hypogonadal men with psoriasis benefit from long-term testosterone replacement therapy - a series of 15 case reports. Andrologia 2016, vol. 48, issue 3, 341-346. [DOI: 10.1111/and.12452].

32. SÁNCHEZ REGAÑA M e UMBERT P. Psoriasis in association with prolactinoma. Br J Dermatol. 2000 Oct;143(4):8647. [PMID: 11069474].

33. SBD. Consenso Brasileiro de Psoríase 2012 - Guias de avaliação e tratamento - Sociedade Brasileira de Dermatologia. -2 ed. Rio de Janeiro.

34. TOOSSI P, et al. Assessment of serum levels of osteopontin, selenium and prolactin in patients with psoriasis compared with controls, and their association with psoriasis severity. Clinical and Experimental Dermatology. 2015 Oct;40(7):741-6. [PMID: 25991399].

35. TUĞRUL AYANOĞLU B, et al. Diminished ovarian reserve in patients with psoriasis. Taiwanese Journal of Obstetrics \& Gynecology. 2018 Apr;57(2):227-230. [PMID: 29673665].

36. WU S, et al. Hormonal factors and risk of psoriasis in women: A cohort study. Acta Derm Venereol 2016; 96: $927-93$. [PMID: 26658522].

37. ZIÓŁKOWSKA E, et al. Psoriasis exacerbation after hormonotherapy in prostate cancer patient-Case report. Rep Pract Oncol Radiother, 2010;15(4):103- 106. [DOI: https://doi.org/10.1016/j.rpor.2010.03.003]. 